\title{
Evaluation of the PAS-Port Proximal Anastomosis System in coronary artery bypass surgery (the EPIC trial)
}

\author{
John D. Puskas, MD, ${ }^{\mathrm{a}}$ Michael E. Halkos, MD, ${ }^{\mathrm{a}}$ Husam Balkhy, MD, ${ }^{\mathrm{b}}$ Michael Caskey, MD, ${ }^{\mathrm{c}}$ \\ Mark Connolly, MD, ${ }^{\mathrm{d}}$ John Crouch, MD, ${ }^{\mathrm{e}}$ Anno Diegeler, MD, PhD, ${ }^{\mathrm{f}}$ Jan Gummert, MD, PhD, ${ }^{\mathrm{g}}$ \\ Wolfgang Harringer, MD, ${ }^{\mathrm{h}}$ Valavanur Subramanian, MD, ${ }_{\mathrm{i}}^{\mathrm{i}}$ Francis Sutter, DO, ${ }^{\mathrm{j}}$ and Klaus Matschke, MD, \\ $\mathrm{PhD},{ }^{\mathrm{k}}$ for the EPIC Trial Investigators
}

\begin{abstract}
Objective: During coronary surgery, proximal vein graft anastomoses have been performed by using an aortic partial occlusion clamp to allow for a hand-sewn anastomosis. The purpose of this multicenter, prospective, randomized trial was to evaluate the efficacy of the PAS-Port device (Cardica, Inc, Redwood City, Calif), which allows an automated proximal anastomosis to be performed without aortic clamping.
\end{abstract}

Methods: Between June 22, 2006, and March 22, 2007, 220 patients requiring coronary artery bypass grafting with at least 2 vein grafts were enrolled. Within each patient, 1 graft was randomly assigned to receive a PAS-Port device, and the other was assigned to receive a hand-sewn anastomosis to the ascending aorta. The primary end point was angiographic patency ( $<50 \%$ stenosis $) 9$ months after surgical intervention. Secondary end points included average time to complete each anastomosis and 9-month freedom from major adverse cardiac events.

\begin{abstract}
Results: One hundred eighty-three patients received matched grafts that were angiographically assessed at 9 months. The 9-month graft patency was $82.0 \%$ (150/183) for hand-sewn and 80.3\% (147/183) for PAS-Port grafts. The patency rate of PAS-Port anastomoses was statistically noninferior to that of hand-sewn anastomoses ( $95 \%$ lower confidence limit for difference, $-7.95 \%)$. The freedom from major adverse cardiac events at 9 months was $97.7 \%$ for PAS-Port (95\% confidence interval, $94.5 \%-99.0 \%$ ) and $98.2 \%$ for hand-sewn $(95 \%$ confidence interval, $95.1 \%-99.3 \%$ ) grafts. The PAS-port device was associated with a $4.6 \pm 3.9-$ minute reduction in anastomotic time compared with that seen with a hand-sewn anastomosis $(P<.001)$.
\end{abstract}

Conclusions: The PAS-Port proximal anastomotic device produces an effective anastomosis with a 9-month patency rate that is comparable with that of a hand-sewn anastomosis. It allows for construction of a proximal anastomosis without aortic clamping and requires less time than a hand-sewn anastomosis.

The traditional approach for coronary artery bypass surgery has been to use the left internal thoracic artery to graft the left anterior descending coronary artery and saphenous vein

\footnotetext{
From the Division of Cardiothoracic Surgery, Emory University School of Medicine, ${ }^{a}$ Atlanta, Georgia; Department of Cardiothoracic and Cardiovascular Surgery, the Wisconsin Heart Hospital, ${ }^{\mathrm{b}}$ Milwaukee, Wis; Scottsdale Healthcare Medical Center, ${ }^{\mathrm{c}}$ Scottsdale, Ariz; the Division of Cardiovascular and Thoracic Surgery, St Michael's Medical Center, ${ }^{\mathrm{d}}$ Newark, NJ; the Department of Cardiovascular and Thoracic Surgery, St Luke's Medical Center, ${ }^{\mathrm{e}}$ Milwaukee, Wis; the Department of Cardiac Surgery, Rhönklinik Bad Neustadt, ${ }^{\mathrm{f}}$ Bad Neustadt, Germany; the Department of Cardiac and Thoracic Surgery, Herzzentrum Jena University, ${ }^{g}$ Jena, Germany; the Department of Cardiac, Thoracic, and Vascular Surgery, Klinik für Herz-, Thorax- und Gefäßchirurgie, Städtisches Klinikum Braunschweig GmbH, ${ }^{\text {h }}$ Hannover, Germany; the Department of Cardiothoracic Surgery, Lenox Hill Hospital, ${ }^{\mathrm{i}}$ New York, NY; the Department of Cardiovascular and Thoracic Surgery, Mainline Heart Health Center, ${ }^{\mathrm{j}}$ Wynnewood, Pa; and the Department of Cardiac Surgery, Herzzentrum Dresden, ${ }^{\mathrm{k}}$ Dresden, Germany.

Disclosures: This US Food and Drug Administration trial was funded entirely by Cardica, Inc, the manufacturer of the PAS-Port System.

Received for publication Nov 2, 2008; revisions received Jan 10, 2009; accepted for publication Feb 2, 2009.

Address for reprints: John D. Puskas, MD, Division of Cardiothoracic Surgery, Emory University School of Medicine, 550 Peachtree St, Crawford Long Hospital, 6th Floor, Medical Office Tower, Atlanta, GA 30308 (E-mail: john.puskas@ emoryhealthcare.org).

J Thorac Cardiovasc Surg 2009;138:125-32

$0022-5223 / \$ 36.00$

Copyright (c) 2009 by The American Association for Thoracic Surgery

doi:10.1016/j.jtcvs.2009.02.017
}

grafts to bypass other diseased vessels. Although arterial revascularization might provide a more durable revascularization strategy, ${ }^{1,2}$ venous conduits are still commonly used. For both venous grafts and arterial grafts not harvested in situ, a proximal anastomosis to the ascending aorta must be constructed in addition to a distal anastomosis to the coronary artery target. This has traditionally required clamping of the aorta to perform a hand-sewn anastomosis. However, manipulation of the aorta with a partial occlusion (side-biting) clamp for off-pump procedures and a crossclamp with or without a side-biting clamp for on-pump procedures can be associated with aortic atheroemboli. ${ }^{3-5}$ Aortic manipulation is independently associated with an increased risk of postoperative stroke. ${ }^{6,7}$

Facilitating devices have been developed to allow for construction of a proximal anastomosis without clamping the aorta. ${ }^{8-10}$ These devices are commonly used during offpump procedures to allow for a no-clamp coronary bypass procedure. However, these devices still require a hand-sewn anastomosis to be performed between the graft and the aorta and are associated with some blood loss. Hand-sewn anastomoses with these facilitating devices can be time-consuming, increasing operative and anesthesia times. Furthermore, 


\section{Abbreviations and Acronyms \\ FDA = US Food and Drug Administration \\ MACE $=$ major adverse cardiac event}

hand-sewn anastomoses are dependent on the particular anatomy of the patient and the skills of the surgeon.

The PAS-Port Proximal Anastomosis System (Cardica, Inc, Redwood City, Calif) was specifically designed to create a consistent anastomosis between a saphenous vein and the aorta during either on- or off-pump coronary bypass surgery. It is a fully integrated, automated system that cuts the aortotomy and attaches the vein graft to the aorta in seconds, producing consistent and reproducible anastomoses independent of surgical technique and skill.

The objective of this prospective, randomized, multicenter trial (the Evaluation of the PAS-Port in Coronary Surgery [EPIC] trial) was to evaluate the safety and efficacy of the PAS-Port System in creating a clampless proximal aortic connection and to compare the patency of PAS-Port anastomoses with that of anastomoses constructed by using a standard hand-sewn technique with an aortic clamp.

\section{MATERIALS AND METHODS}

\section{Compliance, Ethical Review, and Informed Consent}

The study protocol was designed to assess the patency of the index grafts and document any adverse effects related to device use. The study was conducted in accordance with current Good Clinical Practices and the ethical principles of the Declaration of Helsinki. The study protocol and amendments, the subject information/consent form, and any materials used to recruit subjects were submitted to and approved by institutional review boards in the United States or independent ethics committees in Germany (European Union). Before the start of the study, approval was obtained in compliance with the requirements of the Clinical Investigation of Medical Devices for $\mathrm{Hu}$ man Subjects. All patients signed informed consent forms before enrollment.

\section{Study Design}

This study was a prospective, randomized, multicenter trial conducted in 12 hospitals in the European Union and the United States. Two hundred twenty subjects were enrolled with the intention to place at least 2 aortocoronary venous bypass grafts. The revascularization strategy for these grafts was determined preoperatively and confirmed intraoperatively before randomization. After satisfying all intraoperative inclusion criteria, including that both vein segments were within specifications for use with the PAS-Port device, the randomization envelope was opened. The 2 saphenous vein graftcoronary target pairings were identified as grafts 1 and 2 and assigned to one of 2 methods for creation of the anastomosis: the hand-sewn control group or the PAS-Port treatment group. The randomization strategy for assignment of the anastomotic method to the 2 index saphenous vein grafts was conducted by using a random number generator. The randomization envelope contained a 6-digit alphanumeric number that was documented on the case report form. With this information, the surgeon was instructed as to which type of anastomotic technique to use for each of the 2 index grafts.

Both on-pump and off-pump surgical procedures were allowed at the discretion of each investigator/surgeon. Facilitating devices (eg, the Maquet Heartstring, Maquet Cardiovascular LLC, San Jose, Calif, or the Novare Enclose, Novare Surgical Systems, Inc, Cupertino, Calif) were allowed for the execution of the hand-sewn index proximal anastomoses to avoid the need for aortic clamping in subjects undergoing off-pump coronary artery bypass at the surgeon's discretion. Intraoperative graft patency was evaluated based on transit time Doppler blood flow measurements. Patients were asked to return for follow-up visits at 3 and 9 months after the operation. A coronary angiogram was performed at 9 months after the operation to document index graft patency.

\section{Study Population}

The study population consisted of male and female patients, 50 to 85 years of age, who required nonemergency bypass of at least 2 coronary arteries. Patients were required to have an ejection fraction of greater than $30 \%$, as measured by means of ventriculographic analysis, nuclear imaging, or echocardiographic analysis. Patients were required to have native coronary artery stenosis of greater than $70 \%$ in each on the intended index grafts. Exclusion criteria included the following: previous cardiac surgery, dialysis-dependent renal failure, serum creatinine value of greater than $2.3 \mathrm{mg} /$ $\mathrm{dL}$ within 30 days before the operation, need for ongoing immunosuppressive therapy, New York Heart Association class IV heart failure symptoms, presence of systemic infection, preoperative intra-aortic balloon counterpulsation, aspirin allergy, history of thromboembolic disease requiring anticoagulation therapy, cerebrovascular accident within 2 weeks before the operation, and life expectancy of less than 1 year.

\section{Populations for Analysis}

Successfully treated patients who (1) had 2 grafts randomized, (2) completed the 9-month angiogram, and (3) had both index grafts (matched pair) studied angiographically at 9 months and were assessable by the angiographic core laboratory constituted the per-protocol population. The intent-to-treat population consisted of patients who had 2 grafts randomized, regardless of whether the grafts were successfully anastomosed to the aorta. For patency, this included all patients with and without 9-month angiograms. For safety, this included all patients regardless of when the subject's grafts were censored. The as-treated population included all randomized patients regardless of the method used for the proximal anastomosis or whether the anastomotic methods were successful.

\section{Intraoperative Data Collection}

During the surgical procedure, the time to complete the proximal handsewn anastomoses, time to use facilitating devices, and loading and deployment time for the PAS-Port system were recorded. All patients received standard anticoagulation management with heparin and protamine reversal in the operating room.

\section{Surgical Technique}

Any concomitant procedures, as well as any other planned nonindex grafts, were performed at the discretion of the surgeon while adhering to the following general guidelines: (1) partial occlusion clamps were not to be placed on the aorta after the PAS-Port anastomosis was created; (2) all proximal anastomoses were to be completed first before completing any distal anastomoses; and (3) facilitating devices were permitted (eg, the Maquet Heartstring or Novare Enclose) for the hand-sewn anastomosis but were to be used and removed before deployment of the PAS-Port Anastomosis System. Different techniques were permitted and documented and included off-pump coronary artery bypass, beating heart supported with cardiopulmonary bypass, and traditional on-pump coronary artery bypass with cardioplegic arrest. Different saphenous vein harvesting techniques were also documented and included endoscopic techniques, bridging skin incisions, or open continuous incisions.

The PAS-Port device (Figure 1) was designed to allow surgeons to load the bypass graft and rapidly complete an automated anastomosis. This device creates the aortotomy and attaches the vein graft to the aorta by means of an automated mechanism, all housed in a single unit. This mechanism allows for a proximal aortic anastomosis to be completed without aortic clamping. Compared with earlier devices, the PAS-Port system allows the endothelium of the 


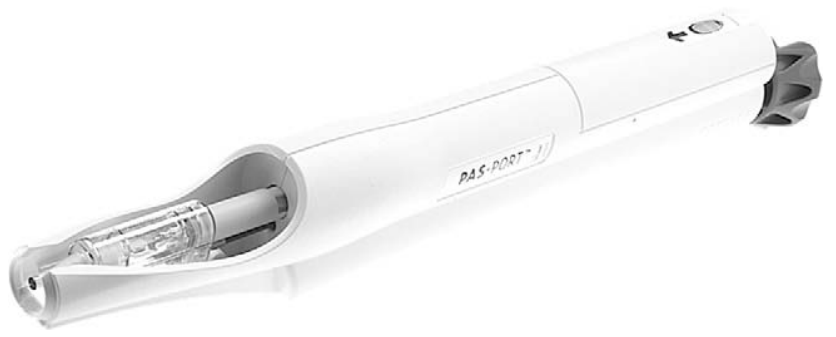

FIGURE 1. PAS-Port device.

vein graft to be untouched during the loading and deployment process. Furthermore, there is no foreign material left within the graft lumen.

\section{End Points}

Primary end points. The primary end point in this study was patency of the proximal anastomoses of the index grafts assessed by means of angiographic analysis at 9 months after the operation in the per-protocol population. In this study patency was defined as less than $50 \%$ stenosis (FitzGibbon A) in the proximal anastomosis of the index graft. ${ }^{11}$

Secondary end points. Patency analyses were also conducted for the intent-to-treat and as-treated populations. In addition, safety analyses were conducted for the intent-to-treat population. The safety analysis included the occurrence and frequency of adverse events at 30 days postoperatively and 9 months. The 9-month freedom from major adverse cardiac events (MACEs) was reported as a Kaplan-Meier curve and compared with the available published literature. MACEs were defined as death, myocardial infarction, or target vessel revascularization associated with either a PAS-Port or hand-sewn index graft. The investigators, the medical monitor, the adjudication committee, and, in instances of subject death, the Data Safety Monitoring Board, were responsible for reviewing all severe adverse events and determining whether they were device related.

The acute procedural success rate was determined for both index grafts in the intent-to-treat population. This rate calculated the percentage of grafts successfully anastomosed by using each method. An unsuccessful anastomosis with either method was defined as one of the following: (1) device or surgical technical failures that resulted in a PAS-Port implant not being successfully placed during the procedure (successful implantation with a second PASPort device was considered a procedural success); (2) a complication that resulted in a PAS-Port implant having to be removed before the subject's discharge; or (3) failure to complete a hand-sewn index graft for any reason.

The time to complete the proximal hand-sewn anastomoses, time to use facilitating devices, and time required to load and deploy the PAS-Port system were recorded. All patients received $81 \mathrm{mg}$ of aspirin daily for the duration of the study. Clopidogrel, $75 \mathrm{mg}$ administered orally, was initiated on postoperative day 1 and continued for 90 days. Additional treatment was administered at the discretion of referring cardiologists.

\section{Angiographic Core Laboratory Analysis}

The primary goal of the analysis by the core laboratory was to independently assess index graft patency at the proximal anastomosis. The core laboratory was blinded to investigative site qualitative analyses and reported patency of the index graft proximal anastomoses according to FitzGibbon criteria. ${ }^{11}$ Based on the protocol definition of patency, a patent proximal anastomosis was defined as an anastomosis classified by the core laboratory as FitzGibbon A. Anastomoses with a FitzGibbon B or FitzGibbon O classification were considered occluded. Grafts considered grade A according to Fitzgibbon criteria had excellent graft flows with unimpaired runoff and proximal anastomotic stenosis of less than $50 \%$. The core laboratory quantitatively determined the amount of proximal anastomosis stenosis by subtracting the minimum internal graft diameter at the proximal anastomosis from the average internal graft diameter approxi-

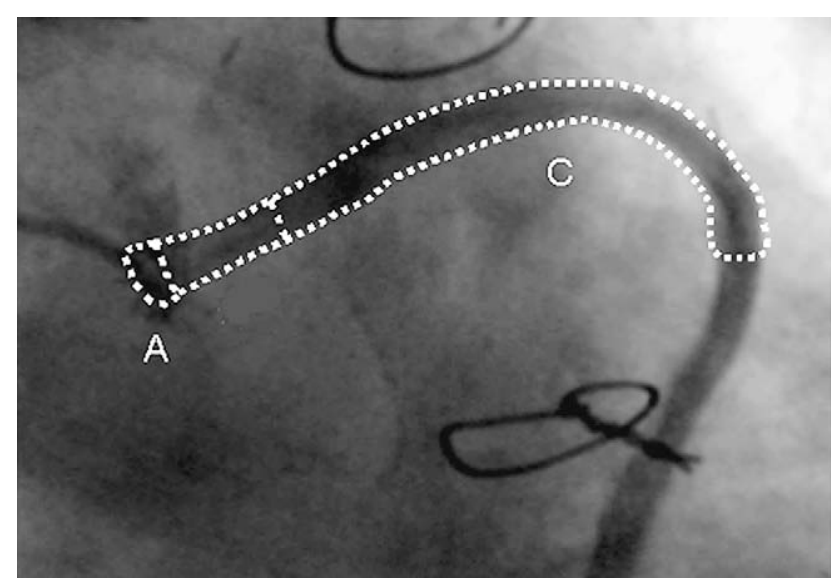

FIGURE 2. $A$, Internal diameter (in millimeters) of the saphenous vein graft at the proximal anastomosis. $C$, Average internal diameter of the graft 2 to $5 \mathrm{~cm}$ distal to proximal anastomosis: Stenosis of proximal anastomosis $(\%)=[(C-A) / C] \times 100$.

mately 2 to $5 \mathrm{~cm}$ distal to the proximal anastomosis and dividing this over the average internal graft diameter approximately 2 to $5 \mathrm{~cm}$ distal to the proximal anastomosis (Figure 2). In addition, the core laboratory reported any abnormalities found in the index grafts or anastomoses, as well as the maximum native stenosis of the target vessel or its tributaries to which the index grafts supplied blood. For subjects unwilling or unable to undergo the protocol-defined 9-month angiogram, efforts were made to obtain multislice computed tomographic angiographic analysis. Because of the limited image resolution of computed tomographic analysis when compared with digital angiographic analysis, assessment of the proximal anastomosis was binary: patent or occluded.

\section{Statistical Methods}

The primary efficacy analysis used the per-protocol population. Only grafts with a matched pair (both a successful PAS-Port and hand-sewn graft in the same patient) were included in this analysis. The primary statistical objective was to test the noninferiority of the 9-month patency rate of the proximal anastomoses performed with the PAS-Port index grafts versus those that were hand-sewn. Noninferiority was defined by a lower limit of the $95 \%$ confidence interval for the success difference (PAS-Port implant minus conventional hand-sewn anastomosis) being greater than $-10 \%$. With a significance level of .05 , a standard proportion of 0.85 , an equivalence limit difference of 0.10 , and a power of $80 \%$, the minimum number of subjects with angiographic follow-up was calculated to be 158 . Assuming that $80 \%$ of the subjects would return for angiographic follow-up, a total of 198 subjects needed to be discharged after undergoing successful bypass with the PAS-Port implant and a hand-sewn index graft. A second primary statistical objective was to provide a $95 \%$ lower confidence limit for the point estimate of the angiographically determined patency rate at 9 months. Specifically, the US Food and Drug Administration (FDA) required that device efficacy would be established only if the lower confidence limit for the point estimate of PAS-Port graft patency at 9 months was at least $70 \%$.

Secondary end points included acute procedural success rate and astreated and intent-to-treat analyses, all of which were evaluated by using inferential statistics without a formal statistical hypothesis. All statistical processing was performed with SAS software (SAS Institute, Inc, Cary, NC).

\section{RESULTS}

This prospective randomized study included 12 investigational sites with enrollment from June 22, 2006, to March 23, 2007; 8 of the sites were located in the United States, 


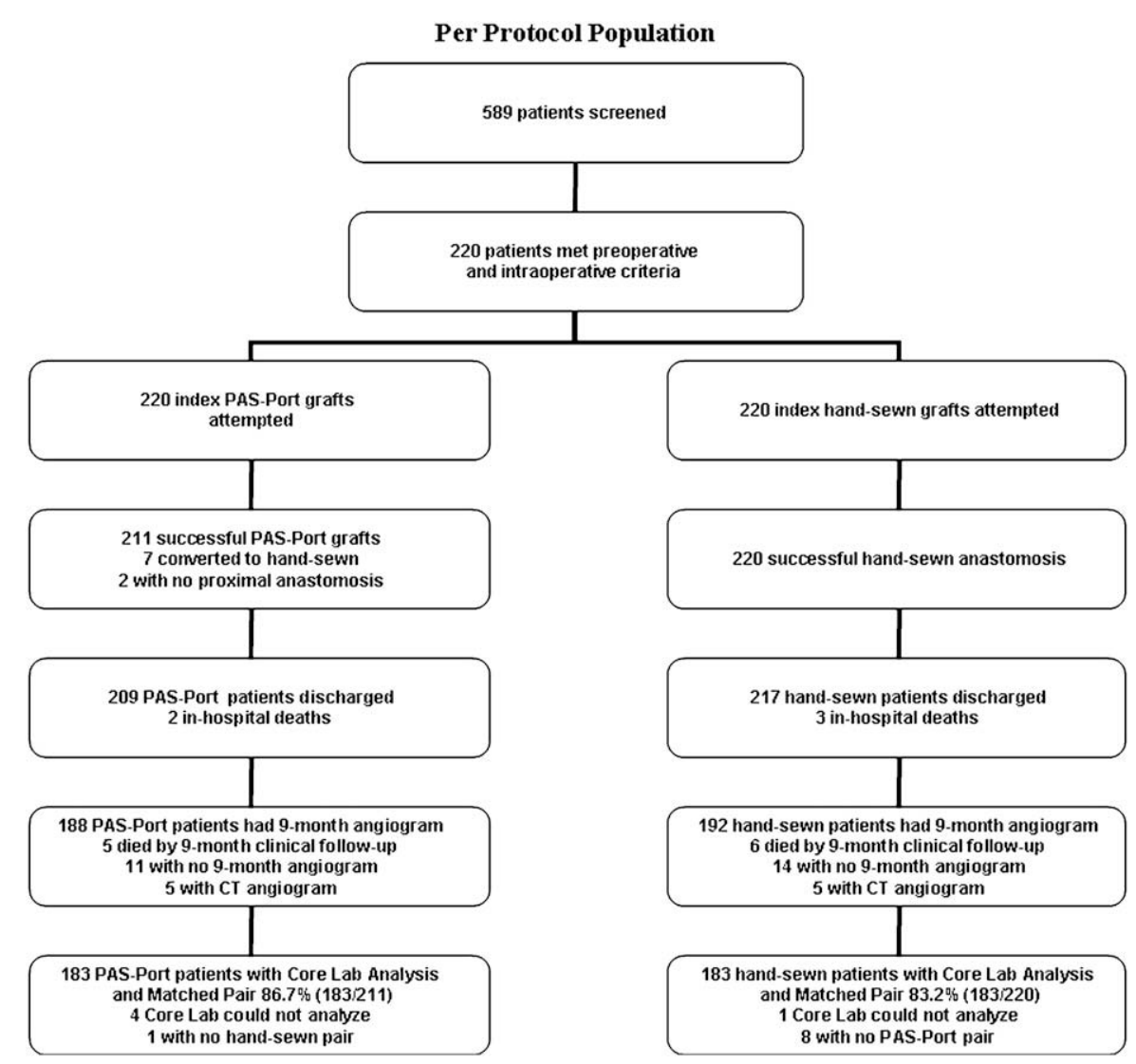

FIGURE 3. Flow diagram of patients in the per-protocol population who had core laboratory angiographic analysis and matched pair comparisons. $C T$, Computed tomography.

and 4 were in the European Union. Five hundred eighty-nine patients were screened for the study, and 249 met the preoperative inclusion and exclusion criteria (Figure 3). Twentynine $(11.7 \%)$ did not meet the intraoperative inclusion criteria and were withdrawn from the study. Patient demographics are presented in Table 1. Of the 220 eligible subjects who participated in the surgical procedure, the PAS-Port system was successfully deployed in 211 (Figure 3 and Table 2). Overall, 188 (85.5\%) of 220 of the patients with PAS-Port anastomoses and $192(87.3 \%)$ of 220 of the patients with hand-sewn anastomoses completed the study with 9-month angiographic follow-up (Figure 3). Because of the way in which the per-protocol group was defined, there were no acute procedural failures and no anastomotic conversions included in this population. Therefore 183 patients met the per-protocol cohort definition, were successfully treated, and had matched anastomosis pairs assessed by the angiography core laboratory.

The graft patency results from the angiographic core laboratory analysis (FitzGibbon Grade A) in the per-protocol cohort demonstrated an average 9-month patency for hand-sewn grafts of $82.0 \%$ (150/183; lower confidence bound, $76.6 \%)$ and $80.3 \%$ (147/183; lower confidence bound, $74.9 \%$ ) for grafts attached to the aorta with the
PAS-Port System. The patency rate of PAS-Port anastomoses was statistically noninferior to the success rate of the hand-sewn anastomoses, with a lower limit of the $95 \%$ confidence interval for the difference between the 2 treatment groups of $-7.95 \%$ (primary objective). The lower confidence bound for the average PAS-Port patency in this cohort was $74.9 \%$, which exceeded the FDA-required minimum lower confidence bound of $70 \%$ (secondary objective). In addition, the acute procedural success rate in the study was $95.9 \%$, which exceeded the target of $90 \%$.

In the intent-to-treat cohort 9-month angiographic patency was comparable in both groups (PAS-Port, 80.6\% [150/186; lower confidence bound, 75.26\%]; hand-sewn, $82.2 \%$ [157/191; lower confidence bound, 77.02\%]). In the as-treated analysis the patency rate was comparable between groups, with an angiographic patency of $80.4 \%$ (148/184; lower confidence, bound $75.0 \%$ ) for PAS-Port grafts versus $82.4 \%(159 / 193$; lower confidence bound, $77.3 \%$ ) for hand-sewn grafts.

\section{Major Adverse Cardiac Events}

Safety analyses were conducted in the intent-to-treat population. The 9-month freedom from MACEs was reported as a Kaplan-Meier curve (Tables 3 and 4) and compared with 
TABLE 1. Patient demographics

\begin{tabular}{|c|c|}
\hline Variable & Total $(\mathbf{n}=\mathbf{2 2 0})$ \\
\hline Age $(y)$, mean \pm SD & $68.1 \pm 7.3$ \\
\hline BMI $\left(\mathrm{kg} / \mathrm{m}^{2}\right)$, mean $\pm \mathrm{SD}$ & $28.8 \pm 4.5$ \\
\hline Male, $\mathrm{n}(\%)$ & $171(77.7)$ \\
\hline History of smoking, n (\%) & $90(40.9)$ \\
\hline Diabetes mellitus, n (\%) & $81(36.8)$ \\
\hline Type I, n (\%) & $2(0.9)$ \\
\hline Type II, n (\%) & $79(35.9)$ \\
\hline Hyperlipidemia, n (\%) & $160(72.7)$ \\
\hline Hypertension, n (\%) & $189(85.9)$ \\
\hline Renal insufficiency, n (\%) & $18(8.2)$ \\
\hline Chronic lung disease, n (\%) & $17(7.7)$ \\
\hline Peripheral vascular disease, $\mathrm{n}(\%)$ & $46(20.9)$ \\
\hline Cerebrovascular accident, n (\%) & $15(6.8)$ \\
\hline Prior myocardial infarction, $\mathrm{n}(\%)$ & $76(34.5)$ \\
\hline Arrhythmia, n (\%) & $20(9.1)$ \\
\hline \multicolumn{2}{|l|}{ CCS class, $\mathrm{n}(\%)$} \\
\hline 0 & $44(20.0)$ \\
\hline I & $35(15.9)$ \\
\hline II & $82(37.3)$ \\
\hline III & $58(26.4)$ \\
\hline IV & $1(0.5)$ \\
\hline \multicolumn{2}{|l|}{ NYHA class, n (\%) } \\
\hline I & $51(23.2)$ \\
\hline II & $110(50.0)$ \\
\hline III & $57(25.9)$ \\
\hline IV & $2(0.9)$ \\
\hline Ejection fraction $(\%$, mean $\pm \mathrm{SD})$ & $56.5 \pm 12.2$ \\
\hline EuroSCORE ( $\%$ chance of mortality) & $3.3 \pm 3.7$ \\
\hline Left internal artery graft use, $\mathrm{n}(\%)$ & $206(93.6)$ \\
\hline Off-pump coronary artery bypass, n (\%) & $140(63.6)$ \\
\hline Cardiopulmonary bypass time $(\mathrm{min})$, mean \pm SD & $100.7 \pm 36.4$ \\
\hline Crossclamp time $(\min )$, mean \pm SD & $63.1 \pm 32.4$ \\
\hline Vein grafts, mean \pm SD & $2.1 \pm 0.4$ \\
\hline Arterial grafts, mean $\pm \mathrm{SD}$ & $1.0 \pm 0.3$ \\
\hline
\end{tabular}

the available published literature. A total of 27 MACEs were recorded, which included 9 deaths (Table 5), 6 myocardial infarctions, and 12 target vessel revascularizations, as reported above. The majority of MACEs were related to target vessel revascularization (12 [44\%]), $4(33 \%)$ of which were possibly related to the use of the PAS-Port system, and $8(67 \%)$ of which were possibly related to the index hand-sewn graft. Three (33\%) of the 9 deaths were possibly related to the use of the PAS-Port system; in none, however, was a clear relationship established. One of the deaths was possibly related to the hand-sewn index graft. Myocardial infarction occurred in 6 subjects, which accounted for $22 \%$ of all MACEs. One of the myocardial infarctions might possibly have been related to the use of the PAS-Port system because the PAS-Port index graft was found to be occluded in the 9-month surveillance angiographic analysis. Four patients had a stroke during the course of the study (2 on-pump and 2 off-pump patients), 3 in the early postoperative period. In all of these patients, an aortic clamp was used for construction of the hand-sewn proximal anastomosis.

The 30-day and 9-month mortality rates in the patients enrolled in the EPIC trial were $1.4 \%$ and $4.1 \%$, respectively. The 30-day and 9-month incidence of myocardial infarction was $1.4 \%$ and $2.7 \%$, respectively. The 30 -day and 9-month incidence of target vessel revascularization was $0.9 \%$ and $2.7 \%$, respectively.

\section{Time Studies}

The time required to load and deploy a PAS-Port device was compared with the time required to complete a handsewn anastomosis (by using the partial occlusion clamp technique or facilitating devices). Significantly less time was required to load and deploy the PAS-Port device compared with side-biting or facilitating hand-sewn anastomoses (Table 6).

\section{Additional Patency Data}

Patency was also defined according to the criteria used for Project of Ex Vivo Vein Graft Engineering via Transfection (PREVENT) IV (patency defined as stenosis, $<75 \%$ ). ${ }^{12}$ Based on these definitions, patency for grafts in the per-protocol cohort was $84.7 \%$ (155/183) with the PAS-Port system and 83.6\% (153/183) for hand-sewn anastomoses. Patency data according to surgical procedure and harvesting technique were also available (Table 7) ${ }^{12}$ and were comparable among the off-pump (83.3\% [246/295]), on-pump with cardioplegic arrest (86.6\% [112/129]), and beatingheart on-pump (84.2\% [19/23]) groups. However, vein graft patency was greater in those patients with veins harvested by using the open technique $(90.8 \%, 130 / 143)$ compared with

TABLE 2. Reasons for PAS-Port failures

\begin{tabular}{lccc}
\hline \multicolumn{1}{c}{ Reasons } & No. & $\begin{array}{c}\text { Total grafts randomized to receive } \\
\text { a PAS-Port (n=220) }\end{array}$ & $\begin{array}{c}\text { Converted to hand-sewn } \\
\text { anastomosis }\end{array}$ \\
\hline Inability to evert vein graft onto implant (not deployed) & 5 & $2.3 \%$ & 4 \\
Implant accidentally dislodged & 2 & $0.9 \%$ & 2 \\
Side branch at implant impeded blood flow and required & 1 & $0.5 \%$ & 1 \\
$\quad$ conversion to hand-sewn anastomosis & & & $0.5 \%$ \\
Graft attached in wrong direction to implant & 1 & $4.1 \%$ & 7 \\
Total & 9 & & 0 \\
\hline
\end{tabular}


TABLE 3. Nine-month freedom from MACE rate by population (Kaplan-Meier estimates)

\begin{tabular}{lccccc}
\hline & \multicolumn{2}{c}{$\mathbf{3}$ mo } & & \multicolumn{2}{c}{$\mathbf{9}$ mo } \\
\cline { 2 - 3 } \cline { 5 - 6 } & Rate & $\mathbf{9 5} \% \mathbf{C I}$ & & Rate & $\mathbf{9 5 \%} \mathbf{C I}$ \\
\hline Intent-to-treat & 94.5 & $90.6-96.9$ & & 91.6 & $86.9-94.6$ \\
Per protocol & 96.7 & $92.8-98.5$ & & 94.9 & $90.4-97.3$ \\
\hline$C I$, Confidence
\end{tabular}

CI, Confidence interval.

those undergoing endoscopic saphenous vein graft harvesting $(79.2 \%, 183 / 231)$.

\section{DISCUSSION}

Proximal vein graft anastomoses to the ascending aorta are a routine part of coronary artery bypass procedures. This has traditionally involved the use of an aortic clamp to allow a bloodless field during construction of the proximal anastomosis. For on-pump coronary procedures, surgical options include performing proximal anastomoses under a single cross-clamp during cardioplegic arrest or placing a partial occlusion clamp after releasing the crossclamp. For off-pump techniques, alternatives include using a partial occlusion clamp or a no-clamp technique with facilitating devices, such as the Maquet Heartstring or Novare Enclose. ${ }^{9,10}$ The impetus for these innovations was to minimize aortic manipulation, which is a well-established risk factor for atheroemboli and stroke. ${ }^{4-7}$ These devices provide a valuable option by allowing a proximal anastomosis to be performed on a suitable portion of the aorta without the need for an aortic clamp. However, these facilitating devices still require the performance of a hand-sewn anastomosis, which might be more technically challenging and involve more blood loss than a conventional anastomosis on a clamped aorta.

Proximal anastomotic connectors were developed to allow for an automated sutureless proximal anastomosis without the use of an aortic clamp. The St Jude Symmetry device (St Jude Medical, Inc, St Paul, Minn) was one of the first devices to gain approval. After a period of initial enthusiasm, ${ }^{13}$ reports emerged documenting early graft stenoses and an unacceptably high incidence of early MACEs with these devices. ${ }^{14-16}$ These results were attributed to excess foreign body within the graft lumen and endothelial damage during loading and deployment. Ultimately, this device was withdrawn from the market in 2004. The Cardica PAS-Port de-

TABLE 4. Nine-month freedom from MACE rate by technique (Kaplan-Meier estimates)

\begin{tabular}{lccccc}
\hline & \multicolumn{2}{c}{ 3 mo } & & \multicolumn{2}{c}{$\mathbf{9}$ mo } \\
\cline { 2 - 3 } \cline { 6 - 6 } & Rate & $\mathbf{9 5 \%} \mathbf{~ C I}$ & & Rate & $\mathbf{9 5 \%} \mathbf{~ C I}$ \\
\hline PAS-Port & 98.2 & $95.2-99.3$ & & 97.7 & $94.5-99.0$ \\
Hand-sewn & 98.6 & $95.8-99.6$ & & 98.2 & $95.1-99.3$ \\
\hline
\end{tabular}

CI, Confidence interval.
TABLE 5. Causes of death for patients not completing the 9-month follow-up

\begin{tabular}{|c|c|c|c|}
\hline Patient & $\begin{array}{c}\text { Time to death } \\
\text { (postoperative } \\
\text { days) } \\
\end{array}$ & Cause of death & $\begin{array}{c}\text { Cardiac- } \\
\text { related } \\
\text { death }\end{array}$ \\
\hline 1 & 56 & Lymphoma & No \\
\hline 2 & 209 & Lung cancer & No \\
\hline 3 & 115 & $\begin{array}{l}\text { Left ventricular } \\
\text { dysfunction after } \\
\text { kinking of hand-sewn } \\
\text { index graft }\end{array}$ & Yes \\
\hline 4 & 33 & ARDS & No \\
\hline 5 & 82 & Myocardial infarct & Yes \\
\hline 6 & 8 & $\begin{array}{l}\text { Left ventricular } \\
\text { dysfunction after early } \\
\text { occlusion of LITA- } \\
\text { LAD bypass as } \\
\text { the major contributor } \\
\text { to subject death }\end{array}$ & Yes \\
\hline 7 & 255 & Pneumonia & No \\
\hline 8 & 9 & $\begin{array}{l}\text { Acute type A aortic } \\
\text { dissection }\end{array}$ & Yes \\
\hline 9 & 2 & $\begin{array}{l}\text { Left ventricular } \\
\text { dysfunction after early } \\
\text { occlusion of LITA- } \\
\text { LAD bypass as } \\
\text { the major contributor } \\
\text { to subject death }\end{array}$ & Yes \\
\hline
\end{tabular}

ARDS, Acute respiratory distress syndrome; LITA, left internal thoracic artery; $L A D$, left anterior descending coronary artery.

vice minimizes the amount of foreign body by limiting the clips to the anastomosis site without exposure to the graft lumen. Similarly, the endothelium is not damaged because it is not exposed to the device during loading and deployment. Early and midterm results of the PAS-Port device have been encouraging, ${ }^{17}$ with comparable patency rates between the PAS-Port and hand-sewn groups. However, larger multicenter studies have not been conducted to compare patency outcomes between these 2 techniques.

In this prospective, randomized, multicenter trial, the 9month patency of venous grafts that received a PAS-Port device was statistically noninferior to those that were hand-sewn. In the intent-to-treat and as-treated analyses,

TABLE 6. Time studies

\begin{tabular}{lr}
\hline \multicolumn{1}{c}{ Anastomosis times by technique } & $\begin{array}{r}\text { Time (min), } \\
\text { mean } \pm \text { SD }\end{array}$ \\
\hline PAS-Port loading and deployment (vs side-biting) & $3.1 \pm 2.5$ \\
Hand-sewn side-biting & $7.7 \pm 2.9$ \\
PAS-Port time savings (vs side-biting)* & $-4.6 \pm 3.9$ \\
PAS-Port loading and deployment (vs facilitating) & $4.1 \pm 2.0$ \\
Hand-sewn facilitating & $9.5 \pm 3.5$ \\
PAS-Port time savings (vs facilitating)* & $-5.5 \pm 3.9$ \\
\hline
\end{tabular}

SD, Standard deviation. $* P<.001$. 
TABLE 7. Patency according to PREVENT IV definitions $(<\mathbf{7 5} \%$ stenosis) ${ }^{12}$

\begin{tabular}{lcc}
\hline & PAS-Port & Hand-sewn \\
\hline Intent-to-treat (angio only) & & \\
Grafts evaluated & 186 & 191 \\
Stenosis $<75 \%$ & $84.9 \%$ & $83.8 \%$ \\
Stenosis $75 \%-99 \%$ & $0 \%$ & $0.5 \%$ \\
Occluded & $15.1 \%$ & $15.7 \%$ \\
\hline
\end{tabular}

results were similar, with no significant difference between the PAS-Port and hand-sewn anastomoses. Furthermore, use of the PAS-Port system resulted in a time savings of approximately 5 minutes compared with the use of an aortic partial occlusion clamp or facilitating device. Vein graft patency in both the PAS-Port and hand-sewn anastomoses was comparable with that seen in other recent studies. ${ }^{12,18-20}$ In our analysis vein graft patency was comparable in the onand off-pump groups ( $86 \%$ vs $83 \%$, respectively).

Use of the PAS-Port device requires a "proximals first" approach to grafting either on or off pump. Nonetheless, the device proved relatively easy to use with a fairly short learning curve. Even though most of the surgeons had not used the device clinically before enrolling patients in the study, the intraoperative rate of successful deployment was $95.9 \%$.

In this trial the 30-day and 9-month mortality rates were $1.4 \%$ and $4.1 \%$, respectively. These mortality rates are comparable with those reported in the recent literature. ${ }^{21-25}$ The 30-day and 9-month myocardial infarction rates observed in the EPIC trial $(1.4 \%$ and $2.7 \%$, respectively) were similar to those cited in the published medical literature ( $2.8 \%$ and $4.9 \%$, respectively) for comparable follow-up periods. ${ }^{12,23,25-27}$

A total of 12 target vessel revascularization procedures were performed in 220 patients with 440 index grafts enrolled in the EPIC trial. Four of the 12 target vessel revascularizations occurred in PAS-Port grafts and were deemed possibly related to the device. By comparison, 8 of the 12 target vessel revascularizations were performed in target vessels revascularized with hand-sewn index grafts. The overall 30-day and 9-month target vessel revascularization rates in this study are $0.9 \%$ and $2.7 \%$, respectively, using the total number of index grafts as the denominator. This is comparable with published reports. ${ }^{12,23,26-28}$ Importantly, 3 early perioperative strokes occurred, all in patients who underwent aortic clamping for the creation of the handsewn graft.

\section{Limitations}

Different surgical and revascularization strategies, such as distal target selection, degree of native coronary stenosis, technique of vein harvest, and off-pump versus on-pump approaches, might influence graft patency. In addition, the individual effect of each treatment (PAS-Port vs handsewn) on MACEs is difficult to assess because each patient received both treatments. However, the MACE rates in the study population as a whole compare favorably with those seen in published reports. Although the primary end point was graft patency (angiographically assessed at 9 months) in the per-protocol population and this analysis included only patients who received both a PAS-Port graft and a hand-sewn graft, these limitations were addressed by the intent-to-treat and as-treated patency analyses. However, smaller degrees of proximal stenosis might have been underestimated with the core laboratory analysis mandated by the FDA.

Another limitation is the heterogeneity of the study groups. This study included patients revascularized with on- and off-pump techniques, simple and sequential grafts, and conventional and facilitating hand-sewn anastomoses. The patency analyses in the hand-sewn cohort were not subdivided into those patients who received a traditional handsewn anastomosis and those who received an anastomosis with one of the facilitating devices (Maquet Heartstring or Novare Enclose). Thus the effect of these different techniques on 9-month patency was not assessed by the core laboratory.

\section{CONCLUSIONS}

In conclusion, the PAS-Port system provides a safe and effective alternative for construction of proximal vein graft anastomoses to the ascending aorta. This device allows an automated and rapid anastomosis with minimal aortic manipulation and can be used in patients undergoing off- or on-pump coronary artery bypass surgery. Nine-month graft patency results are noninferior to the traditional hand-sewn technique. Ultimately, late angiographic follow-up would be required to confirm comparable long-term vein graft patency rates.

We thank our surgical and cardiology colleagues at each of the participating sites who enrolled and cared for patients in this trial.

\section{References}

1. Desai ND, Cohen EA, Naylor CD, Fremes SE. A randomized comparison of radial-artery and saphenous-vein coronary bypass grafts. $N$ Engl J Med. 2004; 351:2302-9.

2. Collins P, Webb CM, Chong CF, Moat NE. Radial artery versus saphenous vein patency randomized trial: five-year angiographic follow-up. Circulation. 2008; 117:2859-64.

3. van der Linden J, Casimir-Ahr H. When do cerebral emboli appear during open heart operations? A transcranial Doppler study. Ann Thorac Surg. 1991;51: 237-41.

4. Blauth CI. Macroemboli and microemboli during cardiopulmonary bypass. Ann Thorac Surg. 2002;74:400-6.

5. Barbut D, Yao FF, Lo YW, Silverman R, Hager DN, Trifiletti RR, et al. Determination of size of aortic emboli and embolic load during coronary artery bypass. Ann Thorac Surg. 1997;63:1262-5.

6. Kapetanakis EI, Stamou SC, Dullum MKC, Hill PC, Haile E, Boyce SW, et al. The impact of aortic manipulation on neurologic outcomes after coronary artery bypass surgery: a risk-adjusted study. Ann Thorac Surg. 2004;78:1564-71. 
7. Calafiore AM, Di Mauro M, Teodori G, Di Giammarco G, Cirmeni S, Contini M, et al. Impact of aortic manipulation on incidence of cerebrovascular accidents after surgical myocardial revascularization. Ann Thorac Surg. 2002;73:1387-93.

8. Guerrieri Wolf L, Abu-Omar Y, Choudhary BP, Pigott D, Taggart DP. Gaseous and solid cerebral microembolization during proximal aortic anastomoses in offpump coronary surgery: the effect of an aortic side-biting clamp and two clampless devices. J Thorac Cardiovasc Surg. 2007;133:485-93.

9. Medalion B, Meirson D, Hauptman E, Sasson L, Schachner A. Initial experience with the Heartstring proximal anastomotic system. J Thorac Cardiovasc Surg. 2004;128:273-7.

10. Akpinar B, Guden M, Sagbas E, Sanisoglu I, Ergenoglu MU, Turkoglu C. Clinical experience with the Novare Enclose II manual proximal anastomotic device during off-pump coronary artery surgery. Eur J Cardiothorac Surg. 2005;27:1070-3.

11. FitzGibbon GM, Burton JR, Leach AJ. Coronary bypass graft fate: angiographic grading of 1400 consecutive grafts early after operation and of 1132 after one year. Circulation. 1978;57:1070-4.

12. Alexander JH, Hafley G, Harrington RA, Peterson ED, Ferguson TB, Lorenz TJ, et al. PREVENT IV Investigators. Efficacy and safety of edifoligide, an E2F transcription factor decoy, for prevention of vein graft failure following coronary artery bypass graft surgery: PREVENT IV: a randomized controlled trial. JAMA. 2005;294:2446-54

13. Carrel T, Englberger L, Keller D, Windecker S, Meier B, Eckstein F. Clinical and angiographic results after mechanical connection for distal anastomosis in coronary surgery. J Thorac Cardiovasc Surg. 2004;127:1632-40.

14. Traverse JH, Mooney MR, Pedersen WR, Madison JD, Flavin TF, Kshettry VR, et al. Clinical, angiographic, and interventional follow-up of patients with aorticsaphenous vein graft connectors. Circulation. 2003;108:452-6.

15. Bergsland J, Hol PK, Lingas PK, Lundblad R, Rein KA, Andersen R, et al. Intraoperative and intermediate-term angiographic results of coronary artery bypass surgery with Symmetry proximal anastomotic device. J Thorac Cardiovasc Surg. 2004;128:718-23.

16. Dewey TM, Crumrine K, Morley AH, Leonard A, Prince SL, Worley C, et al. First-year outcomes of beating heart coronary artery bypass grafting using proximal mechanical connectors. Ann Thorac Surg. 2004;77:1542-9.

17. Kempfert J, Opfermann UT, Richter M, Bossert T, Mohr FW, Gummert JF. Twelve-month patency with the PAS-Port proximal connector device: a single center randomized prospective trial. Ann Thorac Surg. 2008;85:1579-85.
18. Magee MJ, Alexander JH, Hafley G, Ferguson TB, Gibson CM, Harrington RA, et al. Coronary artery bypass graft failure after on-pump and off-pump coronary artery bypass: findings from PREVENT IV. Ann Thorac Surg. 2008;85:494-500.

19. Schwartz L, Kip KE, Frye RL, Alderman EL, Schaff HV, Detre KM. Coronary bypass graft patency in patients with diabetes in the bypass angioplasty revascularization investigation (BARI). Circulation. 2002;106:2652-8.

20. Widimsky P, Straka Z, Stros P, Jirasek K, Dvorak J, Votava J, et al. One-year coronary bypass graft patency: a randomized comparison between off-pump and onpump surgery: angiographic results of the PRAGUE-4 Trial. Circulation. 2004; 110:3418-23.

21. El-Hamamsy I, Cartier R, Demers P, Bouchard D, Pellerin M. Long-term results after systematic off-pump coronary artery bypass graft surgery in 1000 consecutive subjects. Circulation. 2006;114(suppl):I486-91.

22. Hueb W, Soares PR, Gersh BJ, César LA, Luz PL, Puig LB, et al. The medicine, angioplasty, or surgery study (MASS-II): a randomized, controlled clinical trial of three therapeutic strategies for multivessel coronary artery disease: one-year results. J Am Coll Cardiol. 2004;43:1743-51.

23. Goldman S, Zadina K, Moritz T, Ovitt T, Sethi G, Copeland JG, et al. VA Cooperative Study Group \#207/297/364. Long-term patency of saphenous vein and left internal mammary artery grafts after coronary artery bypass surgery: results from a Department of Veterans Affairs cooperative study. J Am Coll Cardiol. 2004;44: 2149-56.

24. Straka Z, Widimsky P, Jirasek K, Stros P, Votava J, Vanek T, et al. Off-pump versus on-pump coronary surgery: final results from a prospective randomized study PRAGUE-4. Ann Thorac Surg. 2004;77:789-93.

25. Hannan EL, Wu C, Walford G, Culliford AT, Gold JP, Smith CR, et al. Drugeluting stents vs. coronary-artery bypass grafting in multivessel coronary disease. $N$ Engl J Med. 2008;358:331-41.

26. Yang JH, Gwon HC, Cho SJ, Hahn JY, Choi JH, Choi SH, et al. Comparison of coronary artery bypass grafting with drug-eluting stent implantation for the treatment of multivessel coronary artery disease. Ann Thorac Surg. 2008;85:65-70.

27. Racz MJ, Hannan EL, Isom W, Subramanian VA, Jones RH, Gold JP, et al. A comparison of short- and long-term outcomes after off-pump and on-pump coronary artery bypass graft surgery with sternotomy. J Am Coll Cardiol. 2004;43:557-64.

28. Unger F, Serruys PW, Yacoub MH, Ilsley C, Paulsen PK, Nielsen TT, et al. Revascularization in multivessel disease: comparison between two-year outcomes of coronary bypass surgery and stenting. J Thorac Cardiovasc Surg. 2003;125:809-20. 